\title{
Preformas para Compósitos Estruturais
}

\author{
Luiz Claudio Pardini \\ Divisão de Materiais, Instituto de Aeronáutica e Espaço, CTA
}

Resumo: Os compósitos estruturais com reforço uni ou bi-direcional, ao contrário dos materiais homogêneos e isotrópicos, apresentam tanto baixa resistência ao cisalhamento interlaminar (<60 MPa) quanto baixa tenacidade à fratura interlaminar. Como decorrência disso estes compósitos são susceptíveis de falha sob baixo carregamento mecânico, e por esse motivo a utilização destes materiais está confinada a componentes estruturais de responsabilidade secundária. A utilização de matrizes com maior tenacidade à fratura em compósitos produz resultados insatisfatórios para aplicações estruturais, pois o ganho na resistência à fratura interlaminar é insuficiente. A utilização de reforços multi-direcionais (preformas) têm gerado resultados significativos nesse particular fazendo com que as pesquisas sejam direcionadas para essa tecnologia. O presente trabalho descreve os métodos que estão sendo utilizados para obtenção de preformas multidirecionais, tanto para aplicação em compósitos poliméricos quanto para compósitos termo-estruturais. A adição de uma fração volumétrica de fibras equivalente a 1 - $2 \%$ na direção normal ao plano do compósito é suficiente para conferir tanto resistência ao cisalhamento quanto tenacidade à fratura interlaminar, sem prejudicar as propriedades originais em outras direções. A utilização de preformas multi-direcionais permite também maior isotropia de propriedades, maior tenacidade à fratura e resistência às tensões térmicas durante o processamento e em serviço.

Palavras-chave: Compósitos estruturais, preformas têxteis, tenacidade à fratura, agulhamento e costura, cisalhamento interlaminar.

\section{Preforms for structural composites}

Abstract: Stacked laminated composites structures (uni and bi-direcional), unlike homogeneous isotropic materials, exhibit low shear strength ( $<60 \mathrm{MPa})$ and low interlaminar fracture thoughness. As a consequence, premature failure during service can occur and their use are restricted mainly to secondary non-load bearing structures. The use of high toughness matrices in composites is not sufficient to improve the interlaminar fracture toughness. Presently, the use of multi-axis preforms have shown significant improvements in interlaminar fracture toughness of composites and a lot of research has been done using this technology. The present work describes methods to obtain multi-axis preforms for either polymer or thermo-structural composites. Many investigations have shown that addition of a 1-2\% fibre volume fraction normal to main fibre plane is enough to provide an adequate shear and interlaminar fracture resistance for the composite keeping in-plane properties. Multi-axis preforms provide higher isotropy, higher fracture toughness and resistance to thermal stresses during processing and service.

Keywords: Structural composites, textile preforms, fracture toughness, needling and stitching, interlaminar shear.

\section{Introdução}

Os compósitos estruturais com matrizes poliméricas são materiais de grande interesse para aplicações em engenharia devido à baixa massa específica $\left(<2 \mathrm{~g} / \mathrm{cm}^{3}\right)$ aliada à alta resistência mecânica
$\left(\sigma_{\mathrm{T}}>500 \mathrm{MPa}\right)^{[1,2]}$. Estes materiais são comumente manufaturados na forma clássica de empilhamento de camadas individuais de tecidos bi-direcionais ou camadas uni-direcionais com orientação definida. $\mathrm{O}$ modo de falha mais comum em compósitos estruturais é a fratura interlaminar, ou delaminação entre as

Luiz Claudio Pardini, Divisão de Materiais, Instituto de Aeronáutica e Espaço, Centro Técnico Aeroespacial, CEP: 12248-904, São José dos Campos, SP. E-mail: Ipardini@iconet.com.br 
camadas. Esse tipo de fratura ocorre mesmo sob baixo carregamento mecânico comprometendo assim a integridade estrutural do componente. Essa limitação restringe as aplicações de compósitos. A solução para suplantar essa deficiência foi obtida pela disposição do reforço de fibras em multi-direções. A resistência à delaminação é medida comumente por meio do ensaio de resistência ao cisalhamento interlaminar, sendo uma medida comparativa e uma forma qualitativa da medida de adesão interfacial. Devido às limitações desse ensaio, tais como a ocorrência de falha não exclusivamente devida à cisalhamento e à impossibilidade de cálculo do módulo em cisalhamento, foi necessário o desenvolvimento de métodos de teste que pudessem resultar em uma medida analítica quantitativa do crescimento da delaminação em compósitos. Um grande esforço nesse sentido foi empreendido por pesquisadores até que o ensaio de tenacidade à fratura interlaminar $\left(\mathrm{G}_{1 \mathrm{C}}\right)$, originalmente desenvolvido para medida de tenacidade de adesivos em metais na década de 60 , fosse adotado como metodologia padrão para compósitos estruturais ${ }^{[3,4]}$.

\section{Compósitos Poliméricos}

Os compósitos poliméricos possuem baixos valores de tenacidade à fratura interlaminar. Por exemplo, valores de $G_{1 C}$ próximos a $150 \mathrm{~J} / \mathrm{m}^{2}$ são típicos para compósitos uni-direcionais com matriz epóxi, e $\sim 300$ $\mathrm{J} / \mathrm{m}^{2}$ para compósitos com matriz de resina bismaleimida ${ }^{[4]}$. Estes valores são baixos comparados aos metais e, sendo assim, os projetistas sempre confinaram a utilização destes materiais somente em estruturas de pouca ou nenhuma responsabilidade estrutural.

Os esforços empreendidos para promover um aumento no valor da tenacidade à fratura interlaminar sempre se concentraram em duas opções básicas :

- incorporação de aditivos tenacificantes à matrizes de fratura frágil, e

- utilização de matrizes com maiores valores de tenacidade à fratura, ou seja, polímeros termoplásticos.

A incorporação de aditivos tenacificantes em matrizes epóxi, como por exemplo as borrachas líquidas de copolímeros de butadieno-acrilonitrila terminadas em carboxila (CTBN) ou terminadas em amina (ATBN), ou mesmo incorporação de diluentes reativos, como por exemplo o fenilglicidiléter (PGE), possibilitaram que os valores de $\mathrm{G}_{1 \mathrm{C}}$ de compósitos manufaturados com estas formulações pudessem atingir
200-250 J/m² ${ }^{2[5]}$. Entretanto, somente compósitos com matrizes de polímero termoplástico atingiram valores de $G_{1 C}$ superiores a $1 \mathrm{~kJ} / \mathrm{m}^{2}$, embora estas apresentem problemas quanto ao processamento e custo relativamente superior às resinas termorrígidas.

\section{Compósitos Termo-estruturais}

No final da década de 1950 a descoberta dos compósitos de carbono reforçado com fibras de carbono (CRFC), formados a partir de uma matriz carbonosa e fibras de carbono, veio colaborar para que o problema da baixa tenacidade à fratura interlaminar, inerente aos compósitos, tivesse uma solução mais definitiva ${ }^{[7]}$. Os compósitos CRFC, com reforço bi-direcional ou uni-direcional, têm resistência ao cisalhamento da ordem de $10 \mathrm{MPa}$, e não há o que fazer no sentido de que a matriz de carbono apresente maior tenacidade à fratura. Matrizes carbonosas têm $\mathrm{G}_{1 \mathrm{C}}$ na faixa de $60-95 \mathrm{~J} / \mathrm{m}^{2}$, valor este significativamente baixo para resistir às tensões térmicas geradas pelos ciclos de processamento (tratamento térmico), e pelos esforços termo-mecânicos a que o material é submetido durante o serviço. Nesse particular, as gargantas de tubeiras de foguete, como mostra esquematicamente a Figura 1, são um exemplo típico onde ocorrem, na região de menor área, altos gradientes de tensões térmicas ${ }^{[6,7]}$. Se estes componentes forem manufaturados pelo empilhamento de camadas de tecidos de fibras de carbono, como mostra o exemplo da Figura 2, é inevitável a ocorrência de descolamento entre camadas.

Esse fato fez com que todo o esforço de pesquisa fosse direcionado para uma solução tecnológica que fizesse com que as fibras de reforço fossem também dispostas na direção perpendicular ao plano das camadas sem, entretanto, penalizar as propriedades nesse

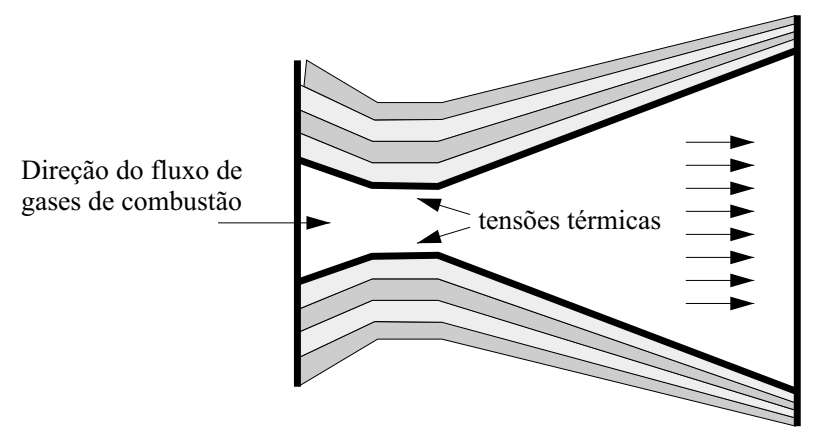

Figura 1. Representação esquemática da região de uma garganta de tubeira de foguete. Áreas escuras representam o tecido de fibras de carbono, e as áreas hachuradas representam a matriz carbonosa. 
plano. Essa nova arquitetura de fibras deu origem a estruturas com reforço multi-direcional, as chamadas preformas ${ }^{[8,9]}$. As preformas (do inglês preform) são definidas aqui genericamente como uma estrutura de fibras de reforço não impregnadas e prontas para moldagem, tendo uma orientação de fibras definida ou aleatória. A preforma é normalmente utilizada com uma geometria pré-definida e adequada ao processamento do compósito. Esse conceito de reforço abriu novas perspectivas aos compósitos fazendo com que aplicações de mais responsabilidade estrutural fossem sendo testadas. O grande empenho empreendido na obtenção de preformas multi-direcionais para compósitos CRFC foram a força motriz do desenvolvimento de estruturas multi-direcionais cujos benefícios se extenderam à todos os demais tipos de compósitos.

\section{Tecidos para preformas}

O processo de tecelagem é uma das mais antigas técnicas de processamento de materiais conhecidas. A forma mais simples e comum de reforço textil para compósitos são os tecidos, mostrados esquematicamente na Figura 2 e consistem de estruturas clássicas bi-direcionais. Os tecidos são produzidos em teares cujas mechas de fibras se entrecruzam em posições mutuamente perpendiculares, alternadas superiores e inferiores, que obedecem a um determinado padrão. Uma orientação bi-direcional é então estabelecida, onde o urdume, que extende-se pelo comprimento do tecido, e a trama são as direções principais. A estrutura bi-direcional dos tecidos é caracterizada pelo espaçamento entre mechas adjacentes, tamanho das mechas, porcentagem de fibras em cada direção, e pelo grau de empacotamento das mechas. Quando os tecidos são submetidos a um esforço mecânico, ocorre uma deformação nas mechas fazendo com que estas apresentem um comprimento efetivo menor ${ }^{[8,9]}$. Além disso, quando um componente manufaturado em compósito é submetido à solicitação mecânica pode ocorrer fricção entre cabos do urdume e da trama, fazendo com que ocorrra uma redução da vida útil do mesmo ${ }^{[8,9]}$.

Os compósitos manufaturados com tecidos bidirecionais, assim como os compósitos manufaturados com camadas individuais de fibras uni-direcionais, apresentam uma região interlaminar que não resiste a esforço de grande magnitude, tendo portanto baixa resistência ao cisalhamento interlaminar $(<100 \mathrm{MPa})$.

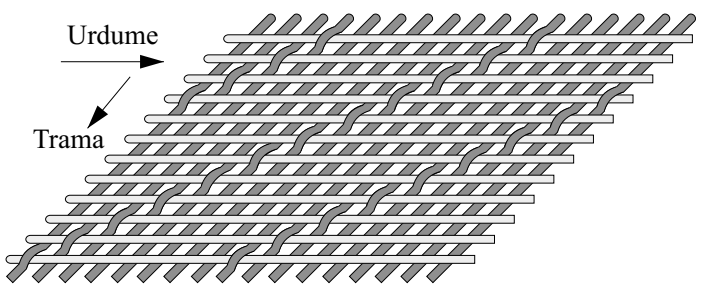

Figura 2. Desenho esquemático de um tecido tipo sarja 8HS com respectivas direções.

Uma das formas encontradas para viabilizar um aumento de tenacidade à fratura interlaminar e a resistência ao cisalhamento interlaminar, é fazer com que a superfície do tecido tenha uma rugosidade superficial inerente. Esse tipo de tela de tecido é obtido pela tecelagem de uma malha sanduíche tri-direcional (3D), que após deixar o equipamento tem os cabos que entrelaçam as duas faces devidamente cortados, conforme mostra esquematicamente a Figura $3^{[10,11]}$. O empilhamento das camadas individuais dá origem a uma preforma com as pontas de cabos em posição interlaminar, em toda a extensão do compósito a ser moldado.

O objetivo do processo é, portanto, fazer com que as regiões entre as lâminas dos tecidos no compósito tenham pontas de cabos de fibras soltas. Estas pontas de cabos de fibras vão fazer com que o reforço tenha uma aparência similar a um veludo ou carpete, conforme mostra a Figura 4. Durante o processo de fra-

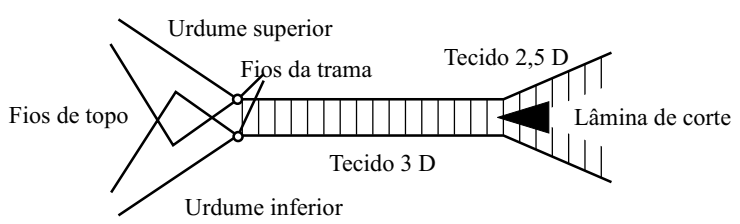

Figura 3. Diagrama esquemático do processo de manufatura de tecidos $2,5 \mathrm{D}^{[16,20]}$.
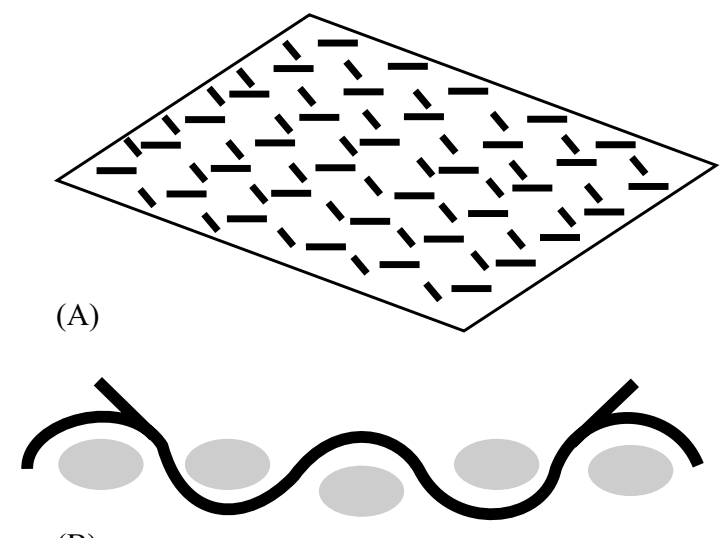

(B)

Figura 4. Vista axonométrica da superfície de um tecido 2,5D (A). Seção transversal do mesmo tecido na direção do urdume (B) ${ }^{[20]}$ 
tura as pontas soltas dos cabos vão interligar duas camadas adjacentes fazendo com que uma energia adicional seja consumida para romper essas ligações. Esse tipo de reforço melhora a tenacidade à fratura interlaminar mas não há aumento relativo nas propriedades perpendiculares ao plano da malha do tecido.

\section{Preformas planas}

$\mathrm{O}$ arranjo multi-direcional de fibras de reforço permite, através de um balanço adequado de orientação e interconecção de cabos de fibras de reforço, a manufatura de compósitos com propriedades praticamente isotrópicas. $\mathrm{O}$ posicionamento de fibras em direções múltiplas permite aumento na tenacidade à fratura devido ao bloqueio da propagação de trincas e também devido a outros processos que consomem energia durante a fratura. Como resultado, o compósito manufaturado com preformas multi-direcionais exibe maior tenacidade à fratura em relação a compósitos com reforço uni e bi-direcional ${ }^{[10]}$. Além disso, a preforma possibilita obter um compósito com dimensões próximas a do componente a ser manufaturado, evitando assim custos adicionais de usinagem.

O estudo dessa interconectividade de cabos de fibras, que dão origem às preformas têxteis, é a força motriz do desenvolvimento de estruturas multidirecionais. As preformas podem ser descritas pelo tipo de fibra utilizada em sua manufatura, pelo número de filamentos em um determinado cabo, pelo tex $(\mathrm{g} / 1000 \mathrm{~m})$ do cabo de fibras, pelo espaçamento entre cabos de fibras, pela fração volumétrica de fibras em cada direção, pelo formato e geometria de tecelagem e pela massa específica aparente. Exemplos clássicos de arquiteturas de preformas são mostrados na Figura $5^{[9]}$.

Embora a questão principal que sempre dominou o desenvolvimento da tecnologia de preformas para compósitos fosse a necessidade de aumentar a resistência interlaminar, outra vertente de pesquisa teve origem no sentido de avaliar o quanto um aumento na resistência ao cisalhamento iria significar uma alteração de propriedades mecânicas em outras direções do compósito.

Os critérios mais importantes para a seleção de uma determinada arquitetura de fibras para compósitos são os seguintes ${ }^{[11]}$ :

(a) reforço multi-axial no plano,

(b) reforço através da espessura,

(c) tomar forma e/ou geometria próximo à geometria final.

Numerosas modificações podem ser obtidas pelo interlaçamento/intrelaçamento de fibras, que vão originar uma multiplicidade de formas que vão posteriormente originar compósitos ${ }^{[11-14]}$. A tenacidade à fratura destes compósitos multi-direcionais vai depender basicamente da direção das fibras em um plano particular de propagação de trinca. $O$ processo de bloqueio de propagação de trincas em compósitos, como por exemplo a delaminação, o descolamento de fibras e outros

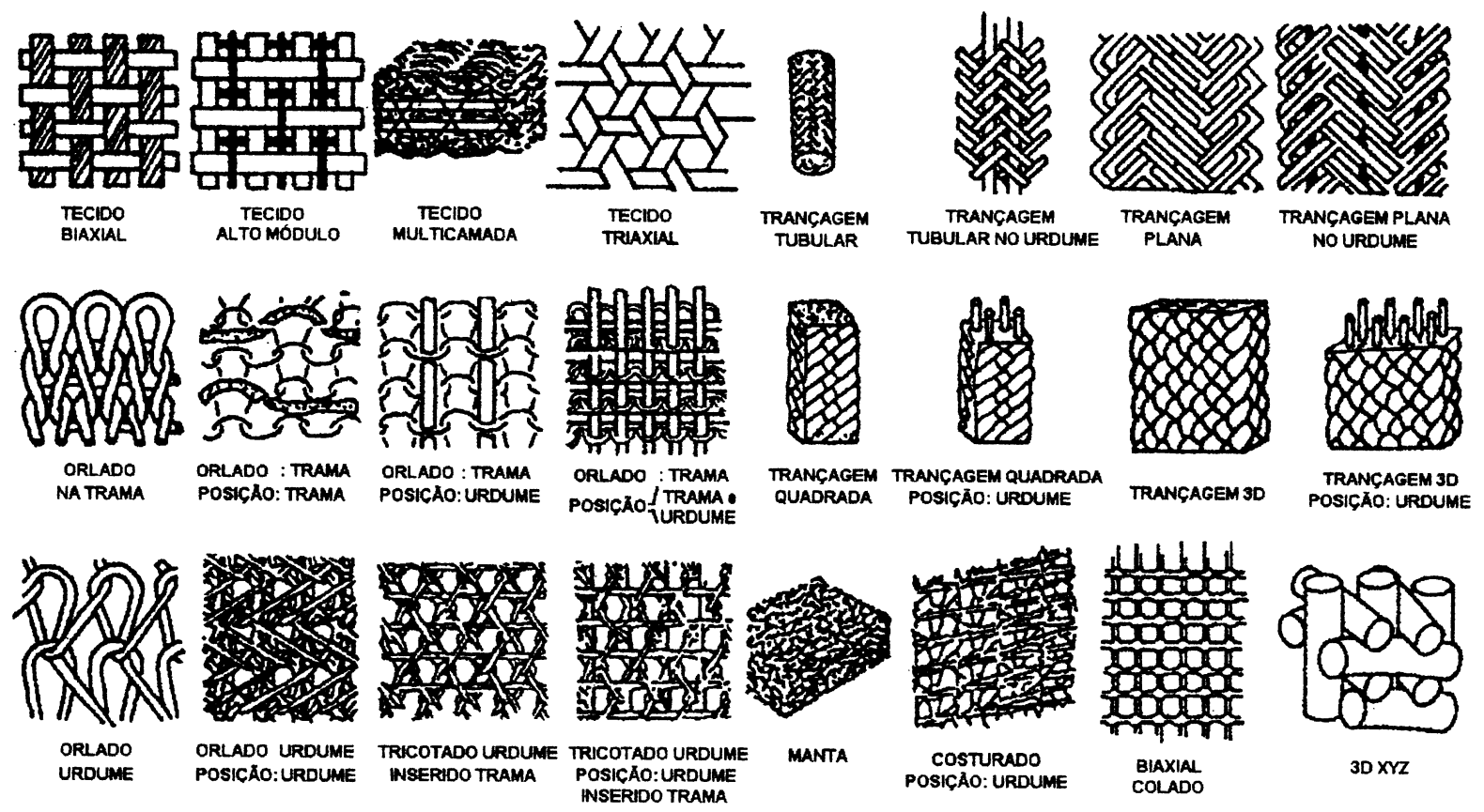

Figura 5. Exemplos típicos de preformas utilizadas na manufatura de compósitos estruturais ${ }^{[9]}$. 
processos que consomem energia durante a fratura conferem ao mesmo tolerância à danos e um modo de falha denominado de pseudo-plástico. Estes compósito vão exibir maior tenacidade à fratura em relação aos compósitos com reforços uni e bi-direcionais ${ }^{[8-11]}$.

\section{Preformas costuradas}

As primeiras soluções utilizadas para promover um aumento na tenacidade à fratura de compósitos poliméricos se concentraram em costurar as camadas individuais, com orientação pré-definida, com filamentos individuais de fibras, conforme mosta esquematicamente a Figura $6^{[6]}$. Esse processo se baseia na inserção de filamentos de fibras individuais através da espessura das camadas de reforço distintas, criando um vínculo entre elas.

Outro método de costura utilizado compreende a utilização de cabos de fibras que também são inseridas através da espessura das camadas individuais do plano. A Figura 7 mostra dois métodos convencionais, costura tipo trava (lock) modificada e costura do tipo corrente (chain) ${ }^{[10]}$. A costura tipo corrente utiliza somente um cabo, enquanto que a costura do tipo trava necessita bobinas e cabos separados. Os parâmetros de costura, que são passíveis de controle, incluem o passo entre as costuras, o espaçamento entre linhas paralelas, o material de costura, e o tex do cabo de costura. Obviamente os cabos vão proporcionar uma resistência interlaminar maior que os filamentos individuais, embora possam apresentam uma dificuldade de inserção através das camadas.

A utilização de fibras individuais ou cabos permite também fazer com que a preforma tenha uma espessura próxima a da peça final evitando assim que seja aplicada compressão mecânica durante a moldagem, que pode danificar o reforço. Nesse caso específico, o processo de moldagem mais indicado

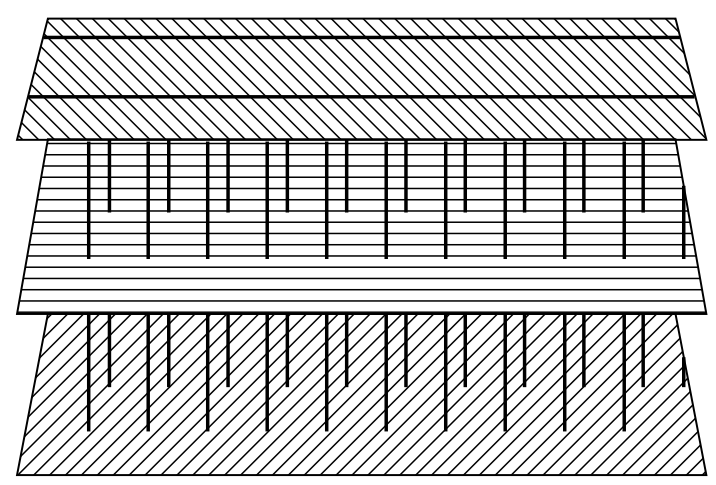

Figura 6. Preforma costurada $+45,0,-45, Z^{[8]}$

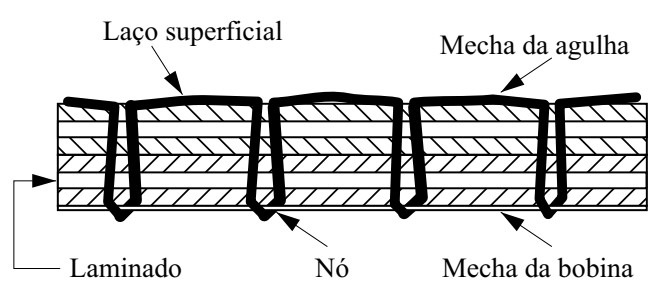

(A)

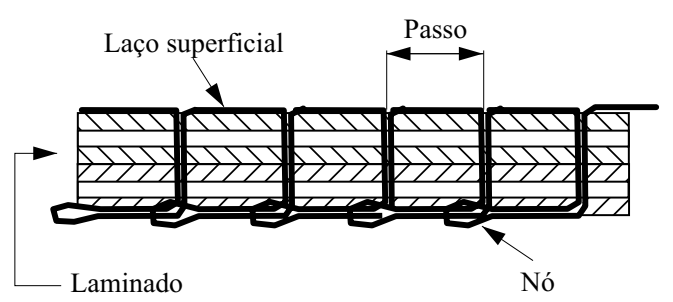

(B)

Figura 7. Tipos de costura utilizada para reforço em preformas, (A) costura tipo trava (lock) modificada; (B) costura tipo corrente (chain) ${ }^{[10]}$.

para obtenção de compósitos é a moldagem por transferência de resina (RTM $)^{[15]}$.

Tanto a orientação quanto a posição da orientação das camadas pode variar. As propriedades no plano para compósitos obtidos com estas preformas são $\sim 30 \%$ superiores às propriedades de tecidos equivalentes, porque os cabos de fibras não apresentam a ondulação inerente dos tecidos. Em grande parte das aplicações, os requisitos de resistência interlaminar e tolerância a danos serão satisfeitos com menos de $\sim 2 \%$ /volume de fibras na forma de costura perpendicular ao plano ${ }^{[16]}$. Além disso, são consideráveis as variações no número de pontos de costura por área que podem ser obtidos, e a tecnologia existente muitas vezes excede os requisitos necessários, não sacrificando assim as propriedades no plano ${ }^{[17-19]}$.

A costura para o tipo de preforma mostrada na Figura 7 é normalmente realizada em equipamentos especialmente projetados para essa finalidade. Um exemplo desse tipo de equipamento é o de camada multiaxial tricotada no urdume (WIMAG), cujo desenho esquemático é mostrado na Figura $8^{[16,19]}$. Equipamentos deste tipo são fornecidos pelas empresas alemãs Liba, Malimo e Mayer. A orientação de fibras nestes equipamentos pode ser livremente ajustada. Além disso, camadas de fibras com orientação aleatória (feltros por exemplo), podem ser adicionadas para formação da preforma. As propriedades no plano para os compósitos produzidos com estas preformas são similares as de compósitos uni-direcionais cujas propriedades na direção correspondente à direção da fibra é cerca de $30 \%$ superior à de um compósito manufaturado com um tecido bi-direcional ${ }^{[19]}$.

Polímeros: Ciência e Tecnologia, vol. 10, № 2, p. 100-109, 2000 


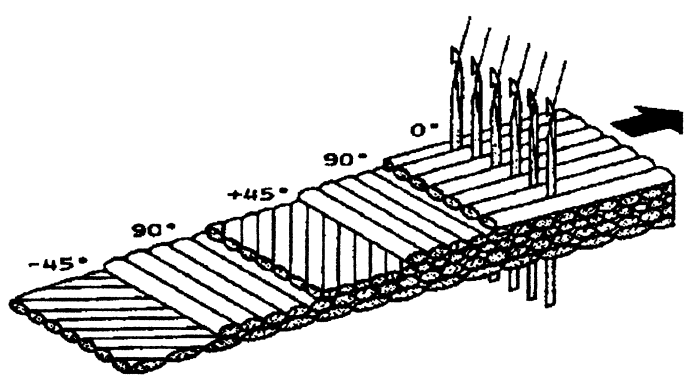

Figure 8. Construção de uma preforma multi-axial costurada na direção do urdume (WIMAG) ${ }^{[16,19]}$

\section{Propriedades mecânicas de compósitos obtidos com} preformas

A tenacidade à fratura interlaminar e a resistência ao cisalhamento são os dois parâmetros básicos de avaliação do quão efetiva foi a inserção de fibras na direção ortogonal ao plano bi-direcional de reforço. A Figura 9 mostra um gráfico com resultados de tenacidade à fratura interlaminar para compósitos costurados. O compósito foi obtido com matriz epóxi e a costura foi realizada com cabos de fibras de aramida, tendo fibras de carbono no plano, com espaçamentos de mecha e regularidade de costura diferentes. Observa-se pelos resultados que a costura de camadas individuais de tecido tem um efeito benéfico no valor de $\mathrm{G}_{1 \mathrm{C}}$, em relação à compósitos uni-direcionais $\left(\mathrm{G}_{1 \mathrm{C}}\right.$ $<0,2 \mathrm{~J} / \mathrm{m}^{2}$ ). A direção da costura em relação à orientação das fibras não altera significativamente o resultado da tenacidade à fratura interlaminar. Entretanto, se o espaçamento entre as costuras for reduzido pela metade ocorre um aumento de $100 \%$ no valor de $\mathrm{G}_{1 \mathrm{C}}$.

Resultados semelhantes foram obtidos com preformas $4 / 5 \mathrm{D}$ que também mostraram que a costura tem influência benéfica quando se considera a resistência ao cisalhamento interlaminar e a tenacidade à fratura interlaminar ${ }^{[21-22]}$. A Figura 10 mostra com-

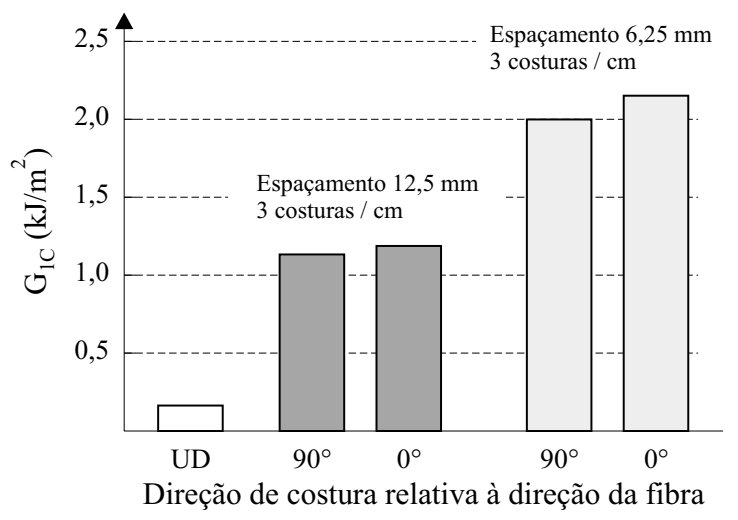

Figura 9. Tenacidade à fratura interlaminar (Modo I) de compósitos costurados com fibra aramida ${ }^{[16]}$. parativamente os resultados de resistência ao cisalhamento interlaminar para compósitos obtidos com uma preforma 4/5D manufaturada com fibras de carbono. Nos respectivos gráficos é mostrada a porcentagem em volume de fibras na direção da costura. O gráfico mostra que após a tensão de cisalhamento máxima o compósito continua a se deformar significativamente. Para efeito de comparação compósitos uni-direcionais de fibras de carbono tem resistência ao cisalhamento de $\sim 60 \mathrm{MPa}$ e falham com redução abrupta da tensão à deflexões de $0,5 \mathrm{~mm}$. Estes resultados indicam que embora não tenha ocorrido um aumento no valor da resistência ao cisalhamento interlaminar, a capacidade do material se deformar aumentou significativamente sem ocorrência de modo de fratura frágil. A redução no valor da resistência ao cisalhamento interlaminar indica que as fibras posicionadas na direção perpendicular ao plano podem estar atuando como concentradores de tensão, induzindo uma redução no valor desta propriedade em relação à compósitos uni-direcionais e bidirecionais.

Por sua vez, resultados obtidos para compósitos manufaturados com preformas de tecidos de fibra de vidro 2,5D, mostrada na Figura 4, mostraram que as pontas de cabos interlaminares favorecem um aumento

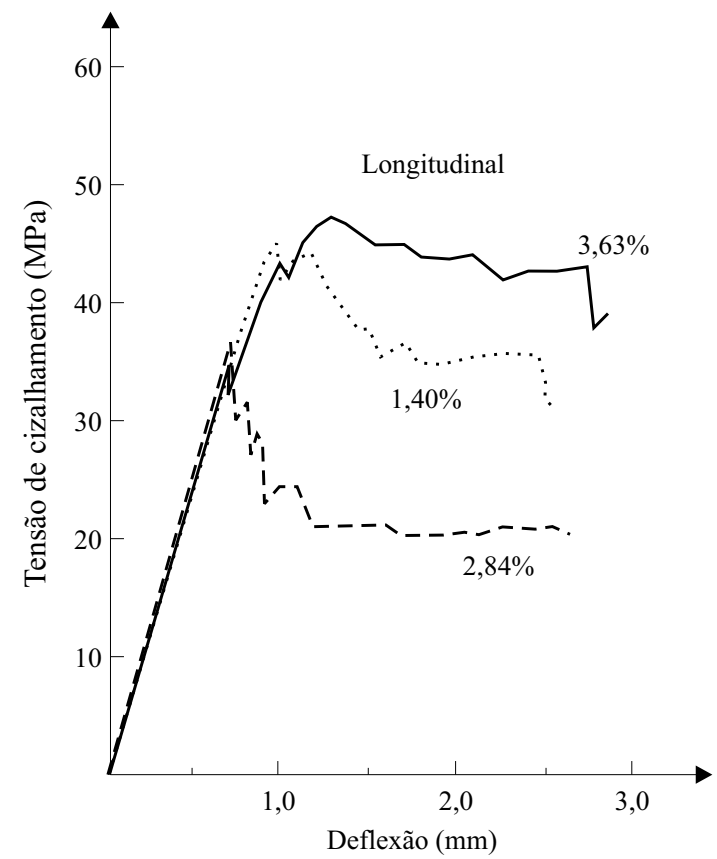

Figura 10. Gráfico típico de tensão de cisalhamento interlaminar em função da deflexão para a preforma 4/5D Aerotiss (fibra de carbono) na direção longitudinal. As porcentagens se referem à direção de reforço perpendicular ao plano de reforço principal. Velocidade de teste: $1 \mathrm{~mm} / \mathrm{min}$. Volume de fibras total $=50 \%{ }^{[22]}$. 
Tabela 1. Tenacidade à fratura interlaminar para compósitos manufaturados com tecidos 2,5D , em função do número de pontas de cabos, e do comprimento destes ${ }^{[16]}$.

\begin{tabular}{ccc}
\hline $\begin{array}{c}\text { Número de pontas } \\
\text { de cabos } \\
(\mathbf{c a b o s} / \mathbf{c m} \mathbf{2})\end{array}$ & $\begin{array}{c}\text { Comprimento } \\
\text { ponta do cabo } \\
(\mathbf{m m})\end{array}$ & $\begin{array}{c}\mathbf{G}_{\mathbf{1 C}} \\
\left(\mathbf{J} / \mathbf{m}^{\mathbf{2}}\right)\end{array}$ \\
\hline Unidirecional & - & 380 \\
3 & 1,5 & 555 \\
3 & 2,5 & 500 \\
3 & 5 & 485 \\
7,7 & 1,5 & 435 \\
7,7 & 2,5 & 550 \\
7,7 & 5 & 660 \\
\hline
\end{tabular}

na tenacidade à fratura interlaminar em relação ao compósito uni-direcional, ver Tabela $1^{[16]}$. Entretanto, um aumento no comprimento da ponta do cabo só favorece um aumento na tenacidade à fratura interlaminar quando um maior número de cabos por área do tecido 2,5D está presente. Os resultados indicam, portanto, que existe um compromisso entre o número de pontas de cabos por área e o comprimento destes no tecido 2,5D. Os resultados obtidos para a tenacidade à fratura interlaminar para esse tipo de configuração de preforma $\left(\mathrm{G}_{1 \mathrm{C}}<660 \mathrm{~J} / \mathrm{m}^{2}\right)$, são entretanto, inferiores aos resultados obtidos com preformas que tenham costura através de toda a espessura do empilhamento $\left(>2 \mathrm{~kJ} / \mathrm{m}^{2}\right)$.

\section{Preformas multi-direcionais e híbridas}

A incorporação de fibras através da espessura, ou em direções múltiplas, é também importante quando tensões térmicas atuam durante o processo de tratamento térmico, como por exemplo, durante o processamento de compósitos CRFC, embora poucos resultados ainda tenham sido reportados na literatura para esses compósitos CRFC.
Tanto as estruturas com requisitos termo-estruturais severos quanto as que necessitam maior isotropia de propriedades podem ser manufaturadas com preformas mais complexas, como as preformas tridirecionais (3D) e tetra-direcionais (4D). Nesse caso específico, os cabos são aglutinados pela utilização do processo de pultrusão, dando origem a varetas com orientação de fibras uni-direcional e seção transversal definida. Além disso, para uma geometria determinada de um componente a ser moldado é necessário projetar uma preforma específica considerando-se os requisitos de propriedades mecânicas finais desejadas. Dessa forma, as propriedades mecânicas são projetadas especificamente para um determinado componente ${ }^{[23]}$. A Tabela 2 mostra, como exemplo, as características para preformas tri-direcionais (3D) manufaturadas com dois tipos de fibras de carbono derivadas de mesofase de piche, Thornel 50 e Thornel 75 (Amoco Co.). As massas específicas aparentes das preformas estão na faixa de 0,65 a $0,70 \mathrm{~g} / \mathrm{cm}^{3}$, e as frações volumétricas de fibras na faixa de 0,09 até $0,14^{[24]}$.

Quanto maior o número de cabos em uma determinada vareta maior seu diâmetro. A fração volumétrica de fibras na vareta também pode ser controlada. Há portanto uma infinidade de possibilidades de arquiteturas 3D e 4D, e a fração volumétrica de fibras pode ser ajustada em direção pré-deteminada. A Figura 11 mostra exemplos de preformas 3D com diferente número de cabos de fibras compondo uma unidade de vareta.

Um exemplo de preforma tetra-direcional (4D) é mostrada na Figura 12. Esta preforma é manufaturada também com unidades de varetas que são dispostas nas diagonais de um cubo. $\mathrm{O}$ ângulo formado entre estas varetas é de $70^{\circ} 30^{\prime}$. A Figura 12B mostra o corte transversal no centro da preforma, onde podese verificar que a célula unitária, constituida pelas quatro diagonais principais, se repete ao longo da geometria definida para o material ${ }^{[25]}$.

Tabela 2. Preformas 3D ortogonais manufaturadas com fibras de carbono ${ }^{[24]}$.

\begin{tabular}{cccccccccc}
\hline \multicolumn{2}{c}{ Massa Específica } & & Número de cabos & & \multicolumn{2}{c}{$\begin{array}{c}\text { Espaçamento entre cabos } \\
(\mathbf{m m})^{(\mathbf{a})}\end{array}$} & \multicolumn{2}{c}{$\begin{array}{c}\text { Volume de fibras } \\
(\boldsymbol{\%})^{(\mathbf{b})}\end{array}$} \\
\hline Material & $\left(\mathrm{g} / \mathrm{cm}^{3}\right)$ & $x$ & $y$ & $z$ & $x, y$ & $z$ & $x$ & $Y$ \\
\hline $\begin{array}{c}\text { Thornel } \\
50\end{array}$ & 0,64 & 1 & 1 & 1 & 0,56 & 0,58 & 0,14 & 0,14 \\
\hline $\begin{array}{c}\text { Thornel } \\
75\end{array}$ & 0,68 & 2 & 2 & 1 & 1,02 & 0,58 & 0,14 & 0,14 \\
\hline
\end{tabular}

a) centro-a-centro, (b) fração volumétrica de fibras em cada direção ortogonal, Thornel 50 ( $\sigma=1,90 \mathrm{GPa}, \mathrm{E}=380 \mathrm{GPa})$, Thornel 75 ( $\sigma=2,1 \mathrm{GPa}$, $\mathrm{E}=520 \mathrm{GPa})$. 


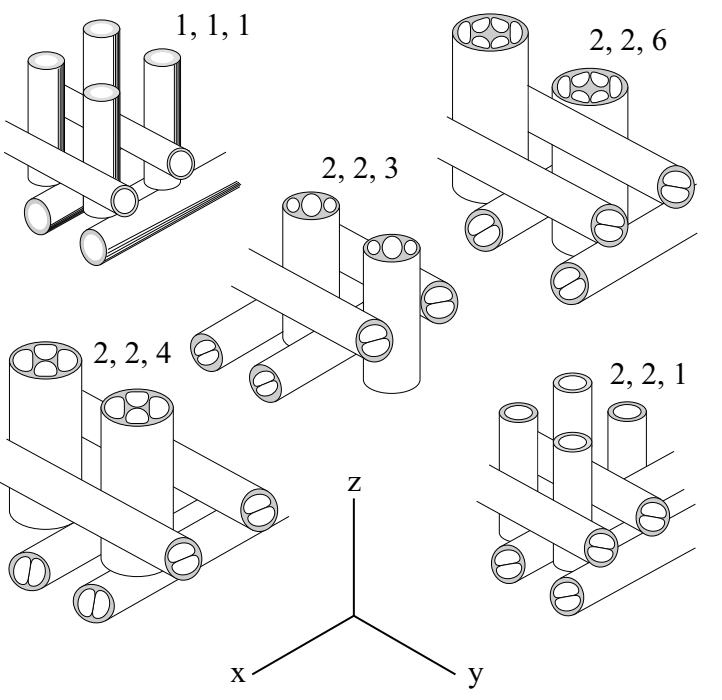

Figura 11. Exemplo de preformas 3D manufaturadas com varetas tendo diferente número de cabos de fibras ${ }^{[24]}$.

Como mencionado anteriormente, quanto maior a complexidade e o projeto de uma preforma maior é o seu custo, e portanto, a utilização de materiais de baixo custo é desejada desde que não sejam comprometidos os requisitos mecânicos estabelecidos no projeto. Nesse particular, as preformas para elementos de fricção de alto desempenho, por exemplo, podem ser manufaturadas pelo agulhamento de fibras curtas de baixo custo, ou agulhamento de tecidos, ou mesmo híbridos agulhados destes ${ }^{[26]}$. O agulhamento de fibras curtas dá origem a feltros. Uma representação típica deste tipo de preforma pode ser vista na Figura 13.

Os feltros são materiais usualmente mais espessos que tecidos convencionais e em princípio são considerados também preformas, tendo orientação aleatória de fibras. Portanto, os feltros agulhados podem substituir na manufatura de compósitos um determinado número de camadas de tecidos, que são de custo relativamente alto. Um feltro agulhado de fibras de carbono de $\sim 5 \mathrm{~mm}$ de espessura e $500 \mathrm{~g} / \mathrm{m}^{2}$ tem custo aproximado de US\$50.00, enquanto que um tecido com tela $8 \mathrm{HS}(\sim 0,25 \mathrm{~mm}$ de espessura) de $300 \mathrm{~g} / \mathrm{m}^{2}$, tem custo aproximado de US\$80.00. A combinação de diferentes tipos de fibras também deve ser considerada. Nesse particular pode-se otimizar propriedades como condutividade térmica, resistência e módulo de elasticidade, simplesmente selecionando um tipo de fibra adequada para cada requisito específico. O agulhamento permite interconectar camadas individuais da preforma, originando assim uma arquitetura próxima à $3 \mathrm{D}$, fazendo com que ocorra uma

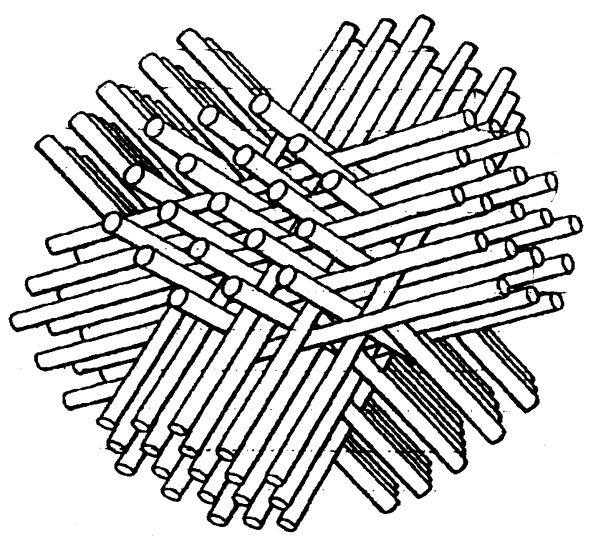

(A)

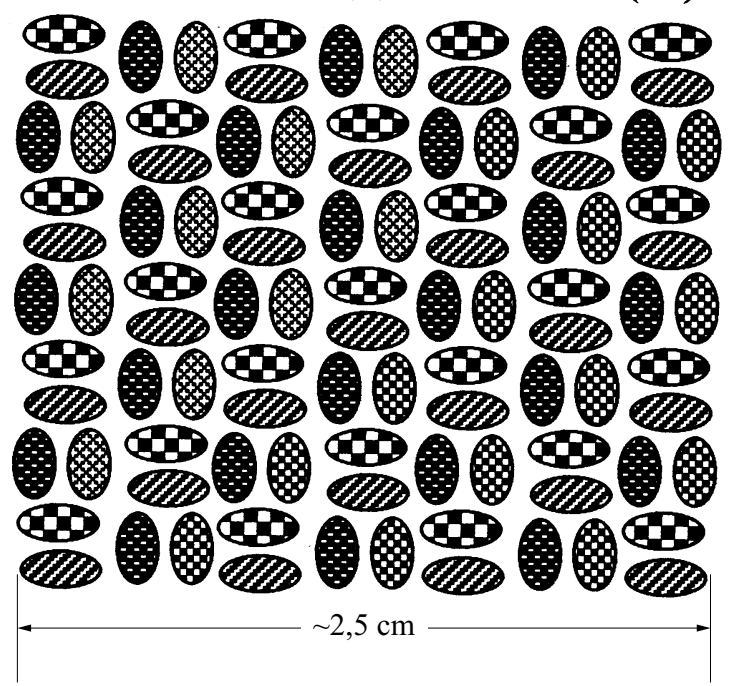

(B)

Figura 12. Desenho esquemático de uma preforma tetra-direcional (4D) ${ }^{[25]}$.

melhora na resistência à delaminação e na tenacidade à fratura em relação à estruturas bi-direcionais.

Só recentemente foram obtidos resultados que fundamentam os benefícios do agulhamento de reforços bi-direcionais para compósitos. Veidt ${ }^{[27]}$ mostrou que a resistência à delaminação $\left(\mathrm{G}_{1 \mathrm{C}}\right)$ de um compósito ma-

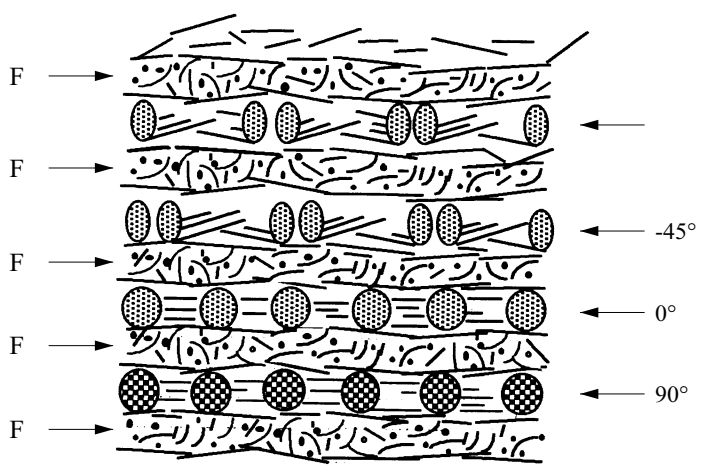

Figura 13. Diagrama esquemático do empilhamento de camadas de tecido e feltro intercaladas ${ }^{[26]}$. 
Tabela 3. Propriedades de um compósito manufaturado com resina epóxi e tecido plain com e sem agulhamento ${ }^{[27]}$.

\begin{tabular}{ccc}
\hline Compósito $\Rightarrow$ & Tecido plain & $\begin{array}{c}\text { Tecido plain agulhado } \\
\mathbf{8 0} \text { agulhamentos } / \mathbf{c m}^{2}\end{array}$ \\
\hline Massa específica $\left(\mathrm{g} / \mathrm{cm}^{3}\right)$ & 2,10 & 1,92 \\
Resistência à Tração $(\mathrm{MPa})$ & 480 & 370 \\
$\mathrm{G}_{1 \mathrm{C}}\left(\mathrm{kJ} / \mathrm{m}^{2}\right)$ & 1,0 & 5,0 \\
\hline
\end{tabular}

nufaturado com tecido agulhado de fibras de vidro e resina epóxi aumenta em cerca de 5 vezes em relação ao compósito manufaturado com o mesmo tecido sem agulhamento. A Tabela 3 mostra os resultados obtidos. Embora ocorra uma redução na resistência à tração de $\sim 20 \%$, no plano, para o compósito agulhado, o módulo de elasticidade praticamente permanece inalterado, enquanto que um aumento de $70 \%$ foi verificado no módulo elasticidade na direção perpendicular ao plano ${ }^{[27]}$. Estes resultados favoráveis indicam tendências que provavelmente podem ser confirmadas para compósitos manufaturados com tecidos de fibras de carbono ou fibras de poliaramida com agulhamento.

\section{Conclusões}

Os compósitos estruturais obtiveram com o advento de preformas multi-direcionais, um ganho significativo na resistência à delaminação e na tenacidade à fratura interlaminar. Os compósitos, obtidos na maioria dos casos como placas finas, podem hoje ser obtidos com espessura considerável tendo resistência ao cisalhamento e à fratura interlaminar adequadas utilizando técnicas de costura, sem contudo perder propriedades no plano de reforço. Com mais essa flexibilidade de orientação de fibras as propriedades dos compósitos estruturais podem se ajustar aos requisitos de projeto.

As preformas multi-direcionais podem também ser manufaturadas com o intuito de conferir isotropia de propriedades, tenacidade à fratura e resistência mecânica em direções pré-determinadas. Estas preformas podem ser obtidas com tamanho próximo à geometria final do componente. Avanços na automatização dos processos para obtenção de preformas multi-direcionais podem contribuir efetivamente para maior produtividade.

\section{Referências Bibliográficas}

1. Matthews, F. L. \& Rawlings, R. D. - "Composite Materials: Engineering and Science", Chapman \& Hall, London, UK (1994).
2. Hull, D. \& Clyne, T. W. - "An Introduction to Composite Materials”, Cambridge, UK (1996).

3. Gibson, R. F. - "Principles of Composite Materials Mechanics", McGraw-Hill, New York (1994).

4. Grady, J. K - Fracture Toughness Testing of Polymer Matrix Composites, in: Handbook of Ceramics and Composites, Nicholas P. Cheremisinoff (ed.), Vol. 2, Mechanical Properties and Specialty Applications, (1993).

5. Riew, C. K. \& Gillham, J. K. - Rubber-Modified Thermoset Resin, ACS 208, American Chemical Society (1984).

6. Brookstein, D. - J. Appl. Polym. Sci.: Appl. Polym. Symp., 47, p. 487 (1991).

7. McEnaney, B. ; Mays, T. - "Relationships between Microstructure and Mechanical Properties in Carbon-Carbon Composites", in Essentials -of Carbon-Carbon Composites, C. R. Thomas (ed.), Royal Society of Chemistry (1993).

8. Klein, A. J. - "Which Weave to Weave", Advance Materials and Processes, 3, p. 40 (1986).

9. Ko, F. K. - "Preform Fiber Architecture for CeramicMatrix Composites", Ceramic Bulletin, 68, n.2, p. 401- (1989).

10. Verpoest, I. "Textile material for composite construction", US Patent 5,271,982 (1993).

11. Efstratiou, V. A. - "Investigation of the Effect of the 2.5D Carbon Fabric Construction on Fabric Reinforced/Polymer Matrix Composite Toughness", Tese de Doutorado, Katholieke Universiteit Leuven, Bélgica (1994).

10. Cox, B. N.\& Flanagan, G. - "Handbook of Analytical Methods for Textile Composites", NASA Contractor Report 4750 (1997).

11. Ko, F. K. - "Three-Dimensional Fabrics for Composites", Chap. 3, in Textile Structural Composites, T. W. Chou \& F. K. Ko (ed.), Composite Materials Series, 3, Elsevier (1989). 
12. McAllister, L. E. \& Lachman, W. L. - "Multidirectional Carbon-Carbon Composites"; in Handbook of Composites, Vol. 4 - Fabrication of Composites - A. Kelly and S. T. Mileiko (1983).

13. McAllister, L. E. - "Multidirectionally Reinforced Carbon/Graphite Matrix Composites", Engineered Materials Handbook, Vol 1:Composites, p. 915, ASM International (1987).

14. Mohamed, M. H. - "Three-dimensional Textiles", American Scientist, 78, p. 530 (1990).

15. Neto, Zabulon S. N. - "Moldagem por Transferência de Resina (RTM), Desenvolvimento do Processo, Fabricação e Avaliação de Laminados", Tese de Mestrado, Instituto Tecnológico de Aeronáutica. (1992).

17. Dexter, H. B. \& Hasko, G. H., "Mechanical properties and damage tolerance of multiaxial warp-knit composites", Composites Science and Technology, 56, p. 367 (1996).

18. Dewalt, P. L. \& Reichard, R. P. - “Just how good are knitted fabrics", J. Reinf. Plast. Comp., V. 13, p. 908, (1994).

19. Du, G. W. \& Ko, F. - "Analysis of multiaxial warpknit preforms for composite reinforcement", Compos. Sci. Techn., 56, p. 253 (1996).

21. Fantino, L. "Complex \& Architectural Textile Preforms - AEROTISS: Potential applications in composite industries" Proc. SAMPE Europe, Paris, p. 185, (1997).

22. Pardini, L. C. "Influência da Costura nas Propriedades em Cisalhamento de Compósitos Estruturais", 13ำ Congresso Brasileiro de Engenharia e Ciência dos Materiais, Curitiba-PR (1998).

23. Tarnopol'skii, U. M.; Zhigun, I. G. \& Polyakov, V. A. - "Spatially Reinforced Composites", Technomic Publishing, Inc., Lancaster (1992).

24. Ngai, T. - "Carbon-Carbon Composites", in International Encyclopedia of Composites, Stuart M. Lee (ed.), VCH Publishers (1991).

25. Pardini, L. C \& Vieira, S. D. - "Preforma Tetradirecional (4D) Vértice-Diagonal para Compósitos Termo-Estruturais", NT-03/AMR/98, CTA/IAE/AMR (1998).

26. Pardini, L. C. - "Controlling mechanical properties on carbon fibre reinforced carbon composites", Anais do 12 Congresso Brasileiro de Ciência e Engenharia de Materiais, Águas de Lindóia-SP, (1996).

27. Veidt, M. - "Stiffness Properties of Three Dimensionally (3D) Reinforced Glass Fabrics Produced by NeedleFelting", Proceedings of ICCM-11, Vol V - Textile Composites and Characterization, p. 742, Gold Coast, Australia (1997).

Recebido: 20/09/99

Aprovado: 06/06/00 